\title{
Correction to: Internet gaming disorder: deficits in functional and structural connectivity in the ventral tegmental area-Accumbens pathway
}

\author{
Ruonan Wang ${ }^{1,2} \cdot{\text { Min } \mathrm{Li}^{1,2} \cdot \text { Meng Zhao }}^{1,2} \cdot$ Dahua $\mathrm{Yu}^{3} \cdot \mathrm{Yu} \mathrm{Hu}{ }^{1,2} \cdot$ Corinde E. Wiers ${ }^{4} \cdot \mathrm{Gene}^{-J a c k}$ Wang ${ }^{4}$. \\ Nora D. Volkow ${ }^{4,5}$. Kai Yuan ${ }^{1,2,3,4,6}$
}

Published online: 5 November 2018

(C) Springer Science+Business Media, LLC, part of Springer Nature 2018

\section{Correction to: Brain Imaging and Behavior (2018)}

https://doi.org/10.1007/s11682-018-9929-6

The original version of this article contained mistakes, and the authors would like to correct them. The correct details are given below:

1. Fig. $2 \mathrm{~b}$, the 9 in the IAT axis of the correlation plot between VTA-right NAc RSFC and IAT should be 90.

2. Fig. 4 d, the "right VTA-NAc mean FA" should be "right VTA-OFC mean FA".

The original article has been corrected.

The online version of the original article can be found at https://doi.org/ 10.1007/s11682-018-9929-6

Kai Yuan

kyuan@xidian.edu.cn

1 School of Life Science and Technology, Xidian University, Xi'an, Shaanxi 710071, People's Republic of China

2 Engineering Research Center of Molecular and Neuro Imaging Ministry of Education, Xi'an, People's Republic of China

3 Inner Mongolia Key Laboratory of Pattern Recognition and Intelligent Image Processing, School of Information Engineering, Inner Mongolia University of Science and Technology, Baotou, Inner Mongolia 014010, People's Republic of China

4 National Institute on Alcoholism and Alcohol Abuse, National Institutes of Health, Bethesda, MD 20892, USA

5 National Institute on Drug Abuse, National Institutes of Health, Bethesda, MD 20892, USA

6 Guangxi Key Laboratory of Multi-Source Information Mining and Security, Guangxi Normal University, Guilin, People's Republic of China 